\title{
Qui est responsable d'un écrit professionnel
}

Journée GERICO, Lille III - Le 5 décembre 1992

\section{Sophie Pène}

\section{(2) OpenEdition}

Journals

Édition électronique

URL : https://journals.openedition.org/edc/2708

DOI : $10.4000 /$ edc. 2708

ISSN : 2101-0366

Éditeur

Université de Lille

\section{Édition imprimée}

Date de publication : 1 décembre 1993

Pagination : 73-87

ISSN : 1270-6841

\section{Référence électronique}

Sophie Pène, "Qui est responsable d'un écrit professionnel », Études de communication [En ligne], Supplément 1993 | 1993, mis en ligne le 30 décembre 2011, consulté le 21 septembre 2021. URL http://journals.openedition.org/edc/2708; DOI : https://doi.org/10.4000/edc.2708

Ce document a été généré automatiquement le 21 septembre 2021.

(c) Tous droits réservés 


\section{Qui est responsable d'un écrit professionnel}

Journée GERICO, Lille III - Le 5 décembre 1992

\section{Sophie Pène}

1 Il est courant de dire : « les écrits professionnels sont sans auteur ». Il est courant aussi de dire l'inverse... C'est un débat possible, sans issue si l'on ne se met pas d'accord sur deux définitions : l'auteur, d'une part, l'écrit professionnel d'autre part...

2 Cette question est donc à contourner.

3 La formulation proposée par Pierre Delcambre : « qui est responsable d'un écrit professionnel ? » permet de dépasser un certain nombre de dichotomies qui nuisent à la problématisation d'un souci qui nous est commun : comment se fait, dans un écrit de travail, l'inscription d'un sujet?

4 Mon but est d'examiner si la notion de responsabilité permet effectivement de lever certains obstacles s'opposant à une définition du sujet de l'écriture professionnelle. Il me semble que la « responsabilité » est une entrée qui dispose de trois atouts essentiels :

- rétablir un continuum entre monde du sujet et coopération avec autrui au travers de la production d'écrits ;

- dégager l'entreprise des approches micro culturelles en montrant la possibilité constante d'une mesure, par la société, et par exemple par un tribunal, de sa responsabilité ;

- mettre en lumière une dimension anthropologique et éthique de l'acte d'écriture. 


\section{La notion de responsabilité : une bonne façon de dessiner le sujet au travail et la prise en charge de ses écrits.}

\subsection{Un premier obstacle : le clivage entre discours concernant l'individu et discours concernant l'organisation.}

Dans les sciences humaines et les sciences de gestion, le monde de l'individu est approché au travers de notions telles que subjectivité, intersubjectivité, place, fonction, rôle. L'organisation, elle, est décrite comme une structure, par exemple en termes de modélisation des flux de documents, de relations fonctionnelles ou opérationnelles.

6 Or, dès qu'on tente de décrire des pratiques d'écriture, on bute sur une difficulté évidente. Il faut bien passer de la sphère individuelle à l'organisation, si tant est qu'on s'intéresse à la construction du sens du document, à sa relation au temps et à l'espace environnant. Et ce ne sont ni les mêmes outils, ni les mêmes notions qui désigneront ce qui dans l'écriture est le fait de la conception, de l'intention, des processus cognitifs ou des choix rédactionnels, et ce qui est le fait de contraintes et de buts, qui propulsent l'écrit dans un espace collectif. Et ce sont en général des visées différentes qui les font prendre en compte: l'individu intéresse d'abord la psychosociologie, l'organisation intéresse d'abord l'économie d'entreprise, le management, les systèmes d'information.

7 Même si la sociologie des organisations a précisément construit les moyens d'un discours articulant individu et organisation, c'est à partir d'un point de vue dominant: celui de l'acteur. L'écrit émane de l'acteur, mais il a une matérialité et une appartenance au monde des objets, qui est d'une autre nature que ce que propose l'interprétation des Systèmes d'Action Concrète (Crozier).

8 La notion de responsabilité permet, me semble-t-il, de penser l'action individuelle comme une donnée de coopération et de force dans l'organisation. Il n'y a pas en effet de responsabilité si l'on ne répond pas à quelqu'un, devant quelqu'un, mais aussi de quelque chose.

\subsection{Le second obstacle est celui de la question de l'auteur et de l' anonyme, ou encore d'auteur anonyme.}

9 L'auteur, si l'on se réfère à l'étymologie augeo, est celui qui augmente, qui fait progresser. Il augmente la confiance, parce qu'il est garant, répondant, autorité et source. Ainsi devient-il modèle et maître.

10 Par là-même il pousse à agir: il est le conseiller, l'instigateur, le promoteur et aussi le créateur. Le terme n'a pris, dans la version latine du mot, auctor, que de façon tardive et mineure le sens d'écrivain-celui qui compose un ouvrage.

A n'en pas douter l'auteur enracine la confiance et la valeur symbolique, mais du côté de l'origine, plutôt que du côté de l'engagement. La notion d'auteur ne suffira donc pas à assurer et à expliquer l'efficience de l'écrit professionnel.

11 Car il n'est certes pas d'écrit au travail qui ait de pouvoir indépendamment d'un auteur, qu'il soit individu ou instance. Mais il n'en est pas non plus qui ait du pouvoir du seul fait 
d'avoir été écrit et diffusé. A la différence d'un auteur littéraire ou journalistique, l'auteur, en entreprise, doit continuer à promouvoir son écrit au-delà du procès d'écriture. Il l'adresse et le diffuse en ambassade, il le défend en réunion, il le glose et le reformule, il le laisse en témoignage, il le livre en texte source amené à produire des paroles et textes seconds.

Par exemple, l'auteur d'un rapport technique proposant le cahier des charges d'une opération de réhabilitation d'un quartier ne pourra pas se dispenser de bien d'autres actions que l'écriture pour persuader autrui du dire vrai de son texte. Par ailleurs, il ne saurait non plus se dispenser d'être auteur...

Ainsi l'auteur éclate-t-il en une multitude de figures (B. Fraenkel), le signataire, voire le double signataire, le fabricant, le commanditaire, le garant, l'expert... Dans l'entreprise, la secrétaire, le demandeur direct, celui qui donne l'ordre de faire, le demandeur indirect, celui qui a besoin d'un texte pour agir, l'auteur partiel, par exemple le juriste, rédacteur des mentions réglementaires, l'informaticien rédacteur des modes de commande, et leur chef, relecteur de l'ensemble, producteur de corrections, obligeant la réécriture, ou le reprographe, le relieur, peuvent à des titres divers se sentir, se dire ou être perçus auteurs du document.

L'auteur n'est donc en entreprise que partiellement identifiable, en tout cas pas avec la solidité et l'homogénéité de l'auteur littéraire, dont l'identité sociale est défendue : sa signature le constitue en auteur, une fois pour toutes, qu'il ait écrit un livre ou dix. La loi le défend contre le plagiat.

Qu'on songe à l'inverse au côté malsonnant qu'aurait l'expression « droits d'auteur » dans le champ professionnel; être dépouillé du contenu de son écrit, entendre ses paroles dans la bouche de sa hiérarchie, relire des fragments d'un de ses rapports dans celui d'un autre ne provoquent d'amertume que chez les salariés juvéniles. Mieux endurci, on comprend que le réemploi d'un texte achève de valider sa vérité et son exploitabilité. Ne pas être plagié est inquiétant pour la carrière : c'est la marque de l'insignifiance et de l'inutilité de son action.

Quant à l'écrit sans auteur, le manuel, le mode d'emploi, il est rare dans mes corpus. S'il apparaît, c'est généralement dans des environnements polémiques :

On a reçu un texte, sans en-tête, si, juste la date, 12 novembre, je crois, et « Le Kremlin », donc c'est bien du siège que c'est parti, et signé « la société » disant : « des locataires ont remarqué le mauvais état des locaux collectifs, il vous est donc demandé..."

Il est vite assimilé au texte de manipulation. Mais il peut relever tout simplement d'un mauvais encodage, d'un rituel mal compris par un néophyte, d'une incertitude quant à la désignation d'un signataire légitime se soldant par une vacance à propos de laquelle l'auteur, se voulant organisateur discret, effacé et pressé, n'imaginera pas les suspicions engendrées.

Les textes organisationnels, les circulaires, les courriers, bien sûr, l'affichage, les notes diverses sont toujours signées... pas forcément, cela s'entend, par leur auteur. Il peut s'agir d'une supposition d'auteur, relevant de cette figure éclatée décrite plus haut, ou d'une substitution franche d'un nom à un autre, pur acte de spoliation justifiée par les liturgies hiérarchiques, ou de la seconde main, (Compagnon, 1979) qu'il s'agisse de citations explicites ou du vaste domaine d'une intertextualité non ressentie : phraséologie, reprises de clauses, de façons de dire, qui peuvent parfois engendrer du non sens, quand les recopies convenues entérinent sans critique des énoncés incohérents. 
La notion d'auteur ne suffit donc pas à recouvrir les façons dont dispose un sujet pour inscrire sa marque dans un texte. L'idée de responsabilité apporte un autre regard, car le « responsable » est celui qui reprend globalement à son compte le déjà dit, qui garantit la cohésion de l'écrit face aux récepteurs.

\subsection{Comment unifier les diverses qualifications de la personnalité au travail?}

19 La personnalité de l'individu au travail peut être abordée par différents biais: celui du caractère, du projet d'action, du passé, de sa place dans l'organisation, de sa compétence d'expert, de sa part dans l'action coopérative...

Ces différentes approches confortent les clivages entre le subjectif et le collectif, le professionnel et le personnel, le vécu, les attitudes, la normativité que véhiculent autant les comportements de travail que les règles organisationnelles.

21 L'idée de responsabilité permet peut-être d'unifier les aspects éthiques, psychologiques et sociaux, comme ceux relevant des savoir-faire, en constituant fermement la personnalité comme l'entité qui rend compte et répond face à autrui de l'action et des principes qui la commandent.

\subsection{La frontière entre l'entreprise et le social : une illusion générée par les organisations.}

L'entreprise est souvent approchée comme une micro société développant ses règles, les créant de façon quasi endogène. Les travaux de Sainsaulieu ne sont pas exempts de cette tendance. Or, si l'entreprise est une société partiellement autonome de son environnement large, comment penser la frontière entre l'entreprise et le social, comment penser les chevauchements entre le social et l'entreprise, les acteurs ne naissant pas comme être sociaux au sein de leur travail?

Les dirigeants des entreprises souhaitent penser celles-ci comme des contenants, des peaux (Anzieu), créatrice d'ordre et d'équilibre, permettant l'action dirigée. D'où la culture d'entreprise, notion si insatisfaisante. Quels que soient les rituels soutenus ou inventés, l'autonomie de cette culture est toute relative; sa stabilité, sa transmission restent bien artificielles; et surtout se pose la question du sens de cette culture.

La notion de responsabilité remet l'entreprise dans un champ de droit commun, rappelant fortement que le rapport de travail est un rapport social, susceptible de transgression, de dommages, de fautes. Ce recours a néanmoins un inconvénient: il contraint à définir négativement la responsabilité, en rendant son exercice manifeste quand elle est engagée dans un conflit voire un délit. C'est une étape indispensable. Elle se justifie. Les exemples de sociétés confrontées à des procès liés à l'écrit ne manquent pas. Il s'agit bien sûr en tout premier lieu de conflits liés au droit du travail, mais, plus généralement, de destinataires portant plainte parce qu'ils s'estiment lésés ou diffamés par les termes d'une lettre. Penser la responsabilité par l'entrée du droit peut nous conduire à l'asseoir plus solidement sur le terrain de l'anthropologique. 


\section{Les deux acceptions juridiques du terme responsabilité.}

24 Au plan juridique la responsabilité se dissocie en responsabilité civile et pénale. A un plan plus général, elle ne peut être pensée indépendamment des domaines éthiques et anthropologiques.

\subsection{La responsabilité civile.}

Pour déterminer l'engagement de la responsabilité civile, le juriste étudie trois questions :

- y a-t-il eu faute?

- cette faute a-t-elle causé un dommage?

- Y a-t-il un lien de causalité entre cette faute et ce dommage ?

Une fois établies les réponses à ces questions, trois types de responsabilités civiles sont dégagées :

- la responsabilité du fait personnel,

- la responsabilité du fait des choses,

- la responsabilité du fait d'autrui, les parents dont un individu est légalement le responsable, les commettants, c'est-à-dire ceux qui sont commis par un individu pour l'exercice d'une tâche.

27 Dans le droit français, l'enquête sur la responsabilité civile repose sur l'idée, positive, que tout dommage est réparable. La recherche des responsabilités est donc la mise en ordre préalable qui permet l'aménagement de la restauration de la personne lésée dans l'état qui précédait le dommage.

\subsection{La responsabilité pénale.}

Elle se différencie de la responsabilité civile en considérant la faute comme un absolu, et non plus du point de vue des droits de la victime.

Elle oblige à répondre des crimes, délits et contraventions devant les juridictions répressives : cour d'assise, tribunal correctionnel, cour d'appel, tribunal de police, cour de sûreté de l'Etat.

\section{Responsabilité et écrit dans l'entreprise.}

Les écrits produits par une entreprise engagent-ils la responsabilité civile ou pénale de leurs auteurs ? Là encore, l'étude est faussée : l'exercice de la responsabilité ne devient phénomène que codé comme délit. C'est au travers d'un cas d'écrit dont le contenu a constitué un délit pénal que, négativement, je vais tenter de placer l'idée de responsabilité d'un écrit dans un cadre plus large que celui de l'écrit interne (privé ?) de l'entreprise, projeté dans l'espace public (Chantraine) par la plainte qu'il a provoqué. 


\subsection{Le délit : une lettre motivant un refus d'attribution de logement.} habitante de Sartrouville. Celle-ci avait sollicité auprès de la société l'attribution d'un logement. L'écart entre l'offre et la demande conduit à rendre beaucoup plus nombreux les refus que les attributions. En soi, refuser un logement est donc un événement courant. Cependant, refuser sans donner de motif n'est pas satisfaisant; commercialement, il importe de donner une raison. Plus profondément, dire " non » sans argument est peu aisé.

La lettre commande le «non, parce que ... ».

Et c'est à ce «non, parce que ... » que débute l'histoire.

Une chargée de gestion de l'agence de Sartrouville écrivit : «non, parce que le quota d'étrangers dans le groupe de logements est atteint ».

Tel est le point d'origine du dossier.

L'habitante de Sartrouville, munie de la lettre, s'adressa au maire, se plaignit du racisme de la société. La mairie ayant semble-t-il quelque contentieux avec la société (conflit de pouvoir dans l'attribution des logements? Volonté de capter un bénéfice de non racisme à ses dépens ?...), aida l'affaire à prendre forme jusqu'au tribunal et jusqu'à la presse.

\subsection{Du fautif au coupable: un condamnation pénale du P.D. G., responsable de ses commettants.}

Il y eut procès, dans lequel furent impliquées, dans une temporaire confusion, la chargée de gestion, son agence à travers la personne du directeur, la société à travers la personne du P.D.G. Le jugement ordonna ainsi les responsabilités : le P.D.G. fut condamné, pénalement, à une amende. On considéra donc que la responsabilité civile n'était pas engagée, la preuve du dommage étant impossible à constituer, car rien n'oblige une société à attribuer de logements dont elle ne dispose pas. Pourquoi la chargée de gestion de gestion n'était-elle pas condamnable ? Commettante de la société, elle existe comme auteur de la lettre, puisqu'elle en est rédactrice et fabricante. Le signataire, et donc l'auteur, le garant, en était le responsable d'agence, distrait, fut-il dit ce jour-là, et donc, fautif, sinon coupable...., n'était pas non plus condamnable : il signe et valide au nom de la société. C'est donc la société qui à rebours se porte garant de ceux qu'elle désigne comme commettants. Et la responsabilité pénale repose sur la contravention constituée par un argument discriminatoire, la notion de quotas ethniques étant évidemment un délit.

\subsection{La responsabilité de l'écrit professionnel se mesure dans un rapport social non clos et non prévisible.}

Que les fautifs ne soient pas coupables place les questions d'auteur et de responsable dans un éclairage assez vif: point d'origine, engagement individuel, la responsabilité se joue bien face à autrui, un autrui qui, au même titre que les figures éclatées des auteurs, se divise et s'instancie diversement, en tout cas selon des modes non prévisibles, d'un espace qui va du domestique au social. Etre responsable impose qu'on puisse répondre à et répondre de à tout moment et à des plans bien différents. 

téléphonique, aurait-elle pu provoquer un tel développement ? Sans doute pas. D'abord parce que la trace orale est plus difficile à constituer en preuve, mais aussi sans doute parce que la puissance symbolique de l'écrit agit avec une autre vigueur, peut-être à cause de ce passage signalé par 0 . Chantraine de «l'espace public à l'espace privé ». Peutêtre aussi parce que l'engagement ou la visibilité du sujet (auteur ? Responsable ? fabricant ?) de la lettre sont d'une autre qualité quand il est question de parole et question de texte.

Soit que le territoire du sujet soit plus facile à circonscrire quand il y a texte et qu'une moindre évanescence ait permis une plainte en retour, soit que d'une façon plus substantielle, mais aussi plus mystérieuse, il soit plus grave d'écrire que de dire et plus pénible, plus cruel, de lire que d'écouter, du moins dans le contexte de ce rapport social particulier et déséquilibré, entre individu et administrations ou sociétés.

\section{La réponse à la condamnation : une réflexion sur la responsabilité.}

38 A l'issue de la publication du jugement, la société a produit un ensemble de documents, intitulé " Dossier Sartrouville ", au moyen duquel elle entendait donner aux salariés les moyens de répondre à des attaques ou critiques portant sur sa politique d'attribution de logements. 

responsabilité: il existe "une grande confusion entre trois logiques qui régissent l'attribution: le droit et les contrats, la logique commerciale, la logique sociale ». Cette formulation montre précisément le lien étroit que la responsabilité implique entre ces trois niveaux de savoir-faire, tous actualisés par les écrits. Si l'on reprend la question de ce qui est dans ce parcours spécifique à l'écrit, on peut peut-être cette fois avancer que l'écrit devient un objet qui exprime des faits et construits des sens engageant non seulement des individus et l'expression de leur savoir ou vouloir faire, mais aussi plus généralement des règles sociales, des modes de pensée et des façons de dire, qui sont régulés par un contrôle tout autant externe qu'interne à l'entreprise. Cette constatation conduit à intégrer la dimension éthique du champ de la responsabilité.

\section{La notion éthique et anthropologique de responsabilité.}

NIETZSCHE (Par-delà bien et ma!), l'apprentissage de la responsabilité se fait en deux temps et dans un double mouvement, celui de l'assujettissement et celui de la liberté du vouloir : la société obtient de l'individu par " un implacable dressage ", " un comportement calculable ». La responsabilité revient à répondre de soi par un assujettissement.

La responsabilité est un concept qui relève du vouloir du sujet, mais en dépassant la limite de la personne pour manifester son enracinement dans des façons de faire instituées, relevant d'une éthique. Car tout vouloir implique un sujet, tout vouloir engage une éthique, et toute éthique s'enracine dans le vouloir d'un responsable. 
47 La responsabilité se construit : de place attribuée, la responsabilité devient une place revendiquée du simple fait de l'initiative. L'individu est « fait » responsable par le groupe qui le domestique. Mais, simultanément, le sujet acquiert le pouvoir de "se faire " responsable, parce qu'il se constitue comme sujet dans le champ du langage, parce qu'il est capable d'évaluation.

Pour revenir au champ très restreint et concret qui nous occupe, il est aisé de constater que cette analyse convient pour rendre compte de l'exemple : l'écrit d'entreprise s'apprend, se duplique, se corrige, moins parce qu'il est objet textuel que parce qu'il doit gagner en conformité avec le modèle : conformité des savoir-faire, mais aussi conformité des façons de les dire, des significations. Et c'est quand la capacité de reproduction atteint une sorte de perfection que l'exercice de la responsabilité individuelle est considéré comme possible.

\subsection{L'attitude de responsabilité.}

"Se faire responsable ", c'est pratiquer l'attitude de responsabilité, hors de tout contrôle. Celle-ci est définie par le fait d'avoir à répondre : il faut donc que quelqu'un soit là, ait à répondre pour. L'exercice de la responsabilité ne peut se passer d'une existence physique, corps ou texte, d'un objet constitué, qui puisse structurer la résistance, le débat, la gestion d'interactions.

Ainsi voit-on dans le dossier dont il est question ci-dessus la façon dont le responsable se constitue par raisonnement régressif, attribuant des gradients de responsabilité à la chargée de gestion, au chef d'agence, au directeur régional, au P.D.G. La légitimité du responsable n'intervient que par détermination mutuelle de l'obligateur et de l'obligé, ce qui suppose qu'ait été explicitées les " places » réciproques, par exemple, dans le cas qui nous occupe, le travail de reprise et d'explicitation des rôles entre le désignataire, l'attributaire, ou encore les jonctions entre discours entrepreneurial et discours juridique, politique de peuplement et d'attribution.

\subsection{Le consentement d'un sujet.}

Autre condition définitoire, le consentement d'un sujet. Si la chargée de gestion dénie pour elle-même le fait d'être responsable, aucune coercition ne peut la constituer comme telle. La responsabilité au travail ne se conjugue pas nécessairement avec la figure de l'auteur: refuser de se sentir auteur d'un texte n'exclut pas qu'on s'en dise le responsable, insérant alors les tâches rédactionnelles dans l'ensemble plus vaste des choses à faire dont on se porte garant.

51 Le consentement à la position de responsabilité se marque par la prise de parole, le fait de répondre. On voit ainsi que peut s'organiser une réflexion sur la part de l'individualité et de la reproduction du modèle dans l'écriture professionnelle, qui serait, d'une certaine façon, une copie lucide et consentie... , éthiquement fort différente d'une copie sans sujet.

\subsection{De quoi répond le responsable?}

On comprend bien les contours flous du champ de l'auteur et de celui du responsable quand on se penche sur le mode de constitution de la responsabilité qu'engendre l'écriture: le procès d'écriture est un engagement pris sur l'avenir ; la capacité à se 
désigner responsable du texte est acquise, dès lors qu'on en est auteur. Se dire subjectivement auteur du texte est une façon d'en anticiper la responsabilité. Etre auteur devient une responsabilité au futur antérieur.

Mais par ailleurs, l'auteur, s'il consent par principe, même implicite, à être responsable de son écrit, ne peut se porter garant des modes de réception de son texte. Il ne peut pour autant se dispenser de chercher à les anticiper. La gestion de la réception relève donc non seulement de la communication (gestion du sens, variation des points de vue, hypothèse sur les stratégies d'autrui) mais aussi du problème anthropologique des images et des effets de responsabilité, en anticipation et en rétroaction.

Le responsable répond donc d'effets qu'il ne peut mesurer autrement que du point de vue de la place qu'il se donne et des assurances dont il dispose sur la pertinence de cette place au sein d'un groupe. C'est ainsi également qu'il peut comprendre quels sont les gradients de responsabilité qui le situent sur l'échelle de la responsabilité assumée collectivement par son entreprise.

55 A la figure du responsable s'opposerait celle de l'irresponsable, décrit par Nietzsche à deux extrêmes de l'axe individu - groupe : l'irresponsable est l'individu réduit à néant par un groupe devenu tout. Mais il peut aussi être soumis à l'exercice d'un vouloir individuel, hors la loi, hors règle, et hors conscience de l'altérité.

La responsabilité ne peut donc se dispenser d'un positionnement à la fois de l'individu au sein du groupe et face à d'autres groupes extérieurs.

\subsection{Les marques discursives de la responsabilité dans l'écriture.}

57 A l'appui de ces remarques, on pourrait esquisser la recherche des marques qu'on attribuerait dans la gestion des discours à la position de responsabilité, par exemple :

- les indicateurs de légitimité individuelle renvoyant à un groupe : le " je », l'exercice du nom propre adjoint au nom de la fonction, l'exercice de la signature personnelle adjoint à la mention du nom imprimé ou à la mention p.o., la marque des initiales de l'auteur (rédacteur du brouillon) et du fabricant (secrétaire) en haut d'un courrier, la situation de l'auteur dans l'en-tête, au milieu d'autres marques d'appartenance (le nom et l'adresse de la société, son logo);

- l'enchâssement du discours dans un mouvement responsif: le courrier, l'ordre de circulation d'un courrier, la liste des récepteurs, les copies, les cahiers de mise à la signature, les formulaires à retourner accompagnant une lettre, les diverses formes de demande d'information ;

- la gestion du procès d'écriture (mode du marquage de l'origine et de l'engagement) selon des modes définis et communs à une entreprise ou à une activité: par exemple les analyses financières, en contrôle de gestion se bâtissent en trois temps: elles s'inaugurent par un constat (parcours de lecture du bilan), à partir duquel l'acteur extrait un diagnostic (construction du sens), qu'il convertit en pronostic ou en recommandation; ou de façon plus générale, toutes les matrices de raisonnement et d'exposition par lesquels une entreprise façonne implicitement ou non le plan de ses écrits. On peut rattacher ces formes à l'exercice de la responsabilité, dans la mesure où l'on s'accorde à y voir un jeu d'acteur prenant place dans des formes codées et déjà données, et évaluant le degré de conformité qu'il souhaite adopter ;

- le consentement et l'être là: le «je», les verbes d'opération de l'esprit (l'appréciation, l'estimation, l'hypothèse); 
- le métadiscursif: l'explication, la justification, la reformulation, les gloses d'un sujet à partir des notions propres à une profession ;

- la capacité à se constituer en discours comme un sujet qui demande, questionne.

\section{Conclusion.}

Faire intervenir la notion de responsabilité dans l'étude des écrits professionnels peut constituer un apport dans quatre directions :

- la question de la responsabilité replace les écrits au sein de l'activité de travail mieux que la question de l'auteur ;

- elle intègre le futur et les effets du travail, au travers de l'engagement;

- elle pose le rapport individu/groupe en le couplant au rapport reproduction et copie/ invention et rédaction, unissant ainsi le sujet de l'écriture aux formes des écrits;

- elle prend en compte les effets sociaux des textes professionnels, au-delà des champs des savoir-faire experts.

Comme la discussion l'a rapidement mis en lumière, elle laisse en suspens la spécificité linguistique de l'écrit et n'est pas forcément propre à l'écrit professionnel, qui se rapproche alors sensiblement du statut de l'écrit journalistique.

\section{BIBLIOGRAPHIE}

Compagnon, A., (1979), La seconde main ou le travail de la citation, Le Seuil.

Fraenkel, B., (1988), Les surprises de la signature, signe écrit, Langage et société, n 44, pp 5-31.

Henriot, J., (1990), La responsabilité, Encyclopedia Universalis.

Jankelevitch, V., (1957), La responsabilité en son for intérieur, in Revue internationale de philosophie, $\mathrm{n}^{\circ} 39$.

Nietzche, F., (1971), Par-delà bien et mal, trad. C. Heim. (Jenseits von Gut und Böse, 1886).

Ricoeur, P., (1949), Philosophie de la volonté. Tome 1: le volontaire et l'involontaire, Paris, Le Seuil.

\section{RÉSUMÉS}

La notion d'auteur d'écriture professionnelle étant problématique l'auteur choisit de la traiter sous l'angle de la responsabilité. A travers une réflexion sur les différents niveaux de la responsabilité (individuellement dans le cadre de l'organisation, juridiquement et éthiquement) l'auteur redéfinit la problématique de l'analyse des écrits professionnels et, plus particulièrement, de l'inscription des sujets dans ceux-ci. La notion de responsabilité a surtout pour avantage, par rapport à celle d'auteur, d'atténuer la place de l'analyse linguistique dans la réflexion sur les écrits professionnels. 
INDEX

Keywords : professional piece of writing, author, responsibility, company, professional identity, law

Mots-clés : écriture professionnelle, auteur, responsabilité, entreprise, identité professionnelle, droit

\section{AUTEUR}

SOPHIE PÈNE

Sophie Pène, IUT, Paris, Département GEA 